\title{
Branchiogenic Basaloid Squamous Cell Carcinoma with Adenosquamous Features. Branchiogenic Carcinoma in the Setting of Another Head and Neck Primary: Literature Review and Report of a Case
}

\author{
Riley E. Alexander, Don-John Summerlin, Muhammad T. Idrees \\ Department of Pathology and Laboratory Medicine, Indiana University School of Medicine, Indianapolis, USA. \\ Email: midrees@iupui.edu
}

Received May $2^{\text {nd }}$, 2012; revised May 27 ${ }^{\text {th }}$, 2012; accepted June $10^{\text {th }}, 2012$

\begin{abstract}
The diagnosis of branchiogenic carcinoma is one of significant controversies in the field of head and neck oncology. Those who support its existence adhere to rigid criteria to validate its existence. Those that deny it, purport that the entity is simply metastatic disease masquerading as a branchial cleft primary. One aspect of agreement between the two opposing views is that a separate head and neck primary disqualifies the diagnosis of branchiogenic carcinoma. We report a case in which branchiogenic carcinoma was diagnosed in the presence of an additional base of tongue primary squamous cell carcinoma based on morphologic dissimilarity and evidence of origination from the basal layer of the branchial cleft epithelium. In doing so, we attempt to make the case that unequivocal histologic evidence of branchial cleft origin is the defining feature of branchiogenic carcinoma and, as with many other carcinomas, should be the diagnostic criterion of choice in issuing the diagnosis. A brief pertinent literature review is presented.
\end{abstract}

Keywords: Adenosquamous; Basaloid Squamous Cell Carcinoma; Branchiogenic Carcinoma; Branchial Cleft; Immunohistochemistry

\section{Introduction}

Branchiogenic carcinoma represents an exceedingly controversial entity within head and neck oncology. Von Volkman first described the entity in 1882 [1]. However, it was not until the mid-twentieth century that the entity gained diagnostic criteria allowing for reproducible classification. Martin et al.'s seminal work on the entity reviewed 250 cases from the literature and an additional 15 cases of their own from Memorial Hospital (now Memorial Sloan-Kettering Cancer Center). From this work, they formulated four criteria to make the diagnosis of branchiogenic carcinoma: 1) the cervical tumor must have occurred somewhere along a line extending from a point just anterior to the tragus of the ear, downward along the anterior border of the sternomastoid muscle to the clavicle; 2) the histologic appearance of the growth must be consistent with an origin from the tissue known to be present in branchial vestigial; 3) the patient must have survived and have been followed by periodic examinations for at least five years without the development of any other lesion that could possibly have been the primary tumor; and 4) the best criterion of all would be the histologic demonstration of a cancer developing in the wall of an epithelial-lined cyst situated in the lateral aspect of the neck [2]. Of note, none of their reviewed cases fulfilled the fourth criteria [2].

In 1989, Khafif et al. reviewed 67 cases [3]. Their work was able to elucidate eight cases that fulfilled Martin et al.'s criteria; however, they reported 14 of the patient had incontrovertible histologic evidence of branchiogenic carcinoma. In addition, they provided two case reports of their own with similar findings [3]. From these findings, they sought to refine Martin's strict criteria by deemphasizing the five-year followup and further emphasizing the following two criteria: 1) the absence of an identifiable primary cancer elsewhere by a thorough evaluation of the patient that included imaging and direct imaging techniques along with pathological examination of biopsy material; and 2) a clear histologic identification of the tumor that included a transition from normal cystic epithelium to dysplasia with transformation to overt malignancy and a significant lymphoid presence that can be ruled out from true nodal tissue by the lack of peripheral lobulation, internodular trabeculae, or perinodular sinuses [3]. 
The work by Khafif et al. essentially refined the criteria set by Martin et al., focusing on two primary aspects: lack of another primary and histologic evidence of transformation of cyst lining to malignancy. Because of this, lack of another primary has become almost an absolutist criterion in diagnosing branchiogenic carcinoma. We report a case where a diagnosis of branchiogenic carcinoma is given in parallel to a diagnosis of squamous cell carcinoma in the base of the tongue. This case introduces the possibility that a second primary is not an absolute contraindication to the diagnosis of branchiogenic carcinoma and that histologic evidence of its origin within a branchial cleft cyst should be included in the diagnostic criteria and carefully considered in clinical evaluation for treatment.

\section{Case Report}

A 64-year-old male presented with a growing mass on the left side of the neck, just inferior to the mandibular arch and near the location of the submandibular glands. Only vague pain was reported with the lesion. The patient reported no prior history of malignancy and there was no recent infectious history. The patient denied any fevers or chills and there was neither significant weight loss nor history of neck irradiation. Exam revealed a nonpulsatile, firm, mobile mass that was nontender to palpation. Computed tomography (CT) of the neck revealed a $2.0 \times 1.5 \mathrm{~cm}$ hypodense lesion in the area of the submandibular gland, adjacent to the jugular vein, and external and internal carotid arteries (Figure 1(A)). An intraoperative frozen section found the lesion to be "suggestive of branchial cleft cyst with atypical epithelial proliferation". In addition to the resection of the mass, neck dissection, with the removal of seven lymph nodes, and biopsies of the left base of tongue and nasopharynx were performed.

On review of the permanent sections of the tissue, the lesion was found to be an encapsulated structure composed primarily of a cyst lined by pseudostratified columnar epithelium (Figures 1(B) and (C)). It was remarkable for atypical and malignant appearing squamous cells arising from the basal layer of the cyst epithelium and was determined to be squamous cell carcinoma (SCC) with apparent branchial cleft cyst present. Adjacent two lymph nodes were also involved, and display partial cystic degeneration.

The base of tongue biopsy revealed squamous epithelium with poorly differentiated squamous cell carcinoma. Importantly, this appeared to be morphologically dissimilar to that found in the primary lesion (Figure 1(D)).

Our institution received these slides for review. Review by our pathologists, including a head and neck

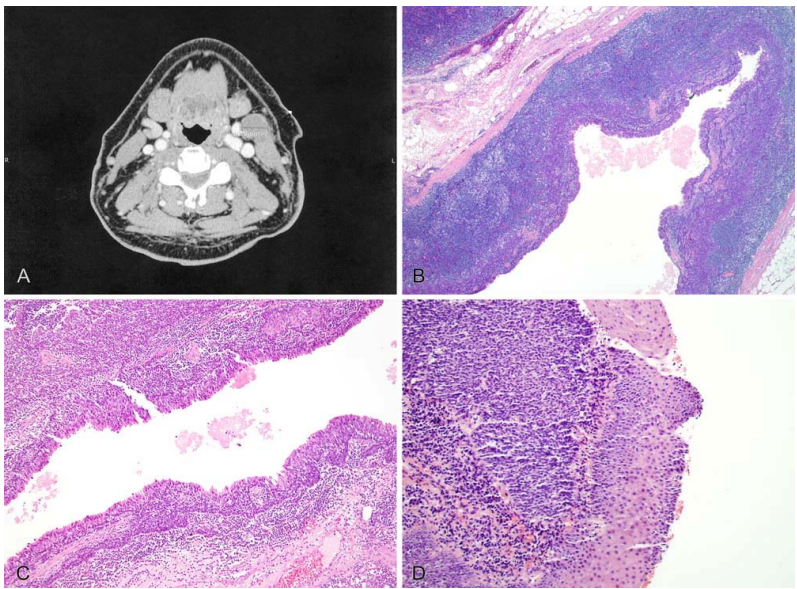

Figure 1. (A) Computed tomography revealing a left-sided hypodense mass measuring $2.1 \mathrm{~cm}$ in greatest dimension in the area of the submandibular gland; (B) Low-power view $(40 \times$, hematoxylin and eosin) of the branchial cleft cyst revealing a pseudostratified columnar epithelium with underlying basaloid squamous-cell carcinoma. A dense lymphoid population of cells is present without well-defined follicles or germinal centers; (C) An intermediate-power view (100×, hematoxylin and eosin) of the branchial cleft epithelium demonstrating a transition from normal pseudostratified columnar epithelium to dysplasia to over malignancy appearing to arise from the basal layer; (D) Highpower view $(200 \times$, hematoxylin and eosin) of the base of tongue biopsy demonstrating a squamous epithelium with adjacent poorly differentiated squamous cell carcinoma that is morphologically distinct from that present in the branchial cleft.

subspecialist, determined the primary lesion to be a branchial cleft cyst with invasive SCC with basaloid and adenosquamous features arising from the cyst epithelium (Figures 2(A)-(D)). The immunohistochemical profile is available in Table 1. Careful review of the primary lesion against that of the base of tongue biopsy was convincing enough to diagnose these as two separate entities: a branchiogenic carcinoma with basaloid and adenosquamous features and a poorly differentiated squamous cell carcinoma in the tongue. Specifically, the branchial cleft cyst showed areas of normal pseudostratified epithelium with transition to dysplastic epithelium and areas of continuity between the basal layer and the infiltrative basaloid squamous cell carcinoma component. Additionally, the lymphoid tissue contained within the branchial cleft cysts was devoid of any follicles or germinal centers and assisted in determination that it was, in fact, a true branchial cleft cyst and not a cystically degenerated node. Two lymph nodes were involved by the branchiogenic carcinoma and the immunohistochemical markers were identical between the adjacent node and the branchiogenic carcinoma (Figures 3(A)-(D)). Unfortunately, due to the paucity of tissue remaining in the tongue biopsy, 
Table 1. Immunohistochemical staining profile of the branchiogenic carcinoma.

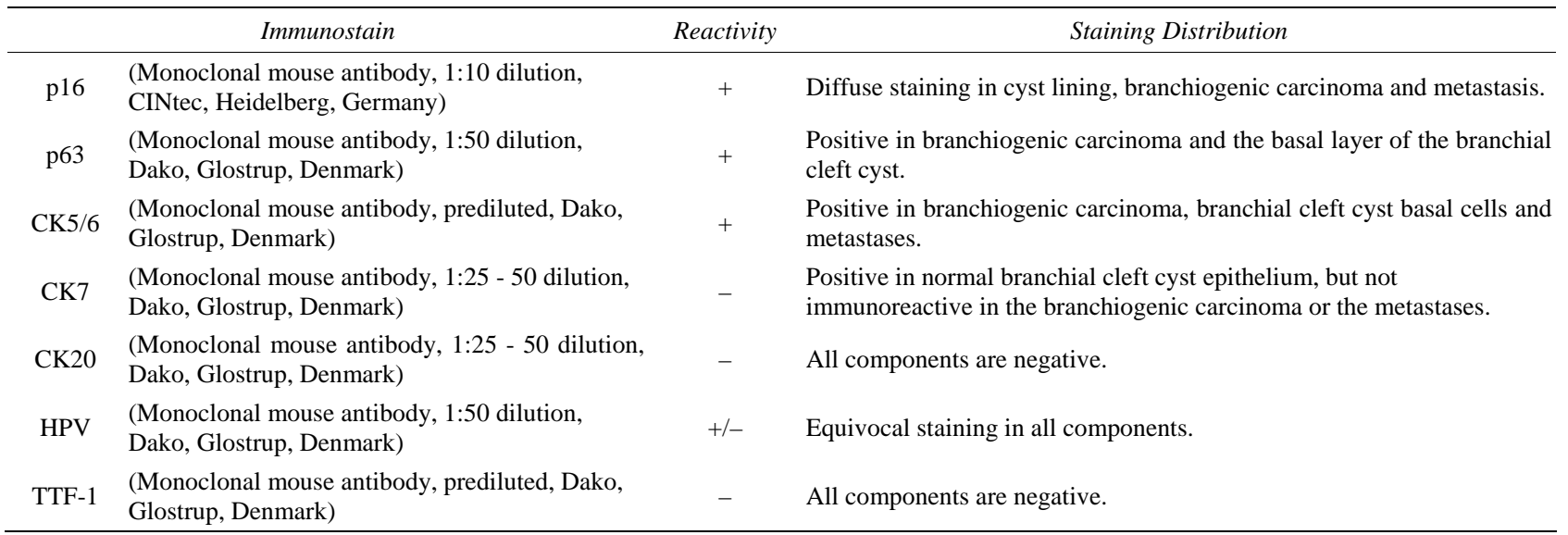

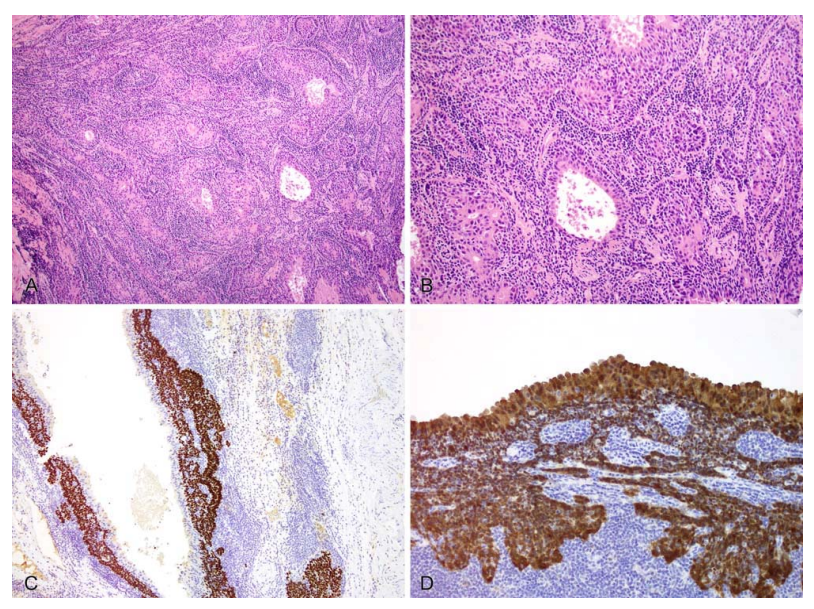

Figure 2. (A) High-power view (200×, hematoxylin and eosin) of the invasive component of the branchiogenic carcinoma, highlighting the basaloid nature of the squamous cell carcinoma; (B) High-power view (200×, hematoxylin and eosin) of the invasive component of the tumor demonstrating both the basaloid and adenosquamous features of this branchiogenic carcinoma; (C) p63 immunostaining of the branchial cleft cyst demonstrating frank positivity in the branchiogenic carcinoma and negativity in the apical layer of the epithelium; (D) p16INK4a immunostaining of the branchial cleft cyst demonstrating immunoreactivity in the epithelium (all layers) and invasive branchiogenic carcinoma.

the entire panel of immunostains was unable to be performed; but, due to the lesion's apparent origin from the basal layer of the branchial cleft cyst epithelium and the morphological dissimilarity between the two, they were signed out as separate primaries.

The patient followed up for treatment at his home institution and no further recurrences are reported to date.

\section{Discussion}

According to the criteria set by Martin and Khafif, this case directly contradicts the hallmark criterion of no

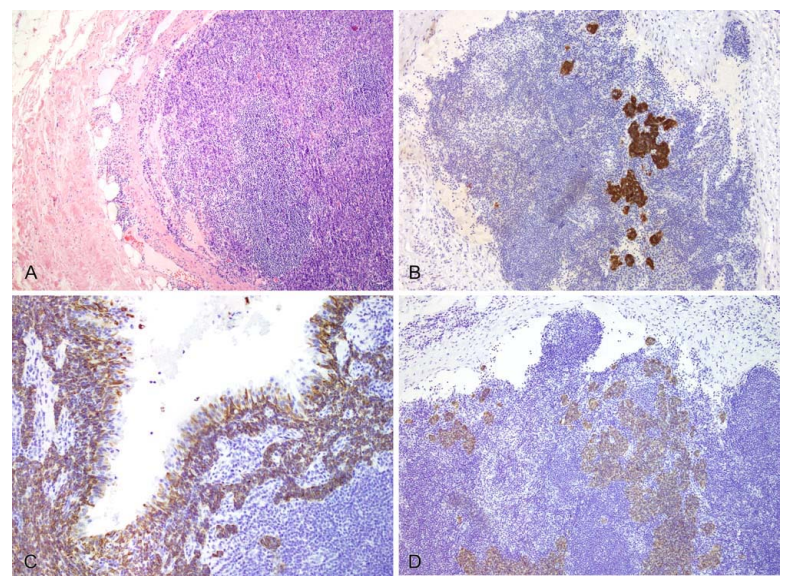

Figure 3. (A) Hematoxylin and eosin view $(40 \times)$ of metastatic deposits of branchiogenic carcinoma within an excised lymph node shown replacing the normal lymphoid tissue; (B) p16 immunostaining $(200 \times)$ highlighting metastatic branchiogenic carcinoma surrounded by non-staining lymphoid tissue in an involved lymph node; (C) CK5/6 immunostaining $(200 \times)$ within the branchial cleft cysts with immunoreactivity of the basal layer of epithelium and invasive branchiogenic carcinoma. Notice negativity of the apical layer of epithelium; (D) CK5/6 immunostaining $(100 \times)$ of involved lymph node showing positivity in areas of involvement by branchiogenic carcinoma.

identifiable second primary. In fact, this case, to some, provides further evidence that the true existence of branchiogenic carcinoma has not been demonstrated $[4,5]$. Thompson and Heffner reviewed 136 cases of cystic SCC of the neck and, using their criteria, identified no true branchiogenic carcinoma. Additionally, they asserted that nearly all branchiogenic carcinomas represented cystic degeneration of a lymph node recapitulating the tonsillar primary. Of important consideration to this case is their belief that those with a "base of tongue" primary are actually tonsillar in origin as well [4]. In examining their five criteria, this case would certainly be 
classified as "atypical", as per their report. Jerezcek-Fossa et al., however, admit the possibility of a branchiogenic carcinoma, yet insist that its rarity precludes a definitive prognostic differentiation from cystic SCC [5].

Recently though, more reports are asserting the diagnosis of a branchiogenic carcinoma as the true diagnostic entity and have tended to use the modified criteria of Khafif [6-9]. These reports have further subdivided branchiogenic carcinoma into those arising within a first or second branchial cleft cyst. However, treatment and prognosis does not vary $[6,8,9]$.

The expression of p16INK4A has proven useful in differentiating benign branchial cleft cysts from metastatic oropharyngeal cancer [10]. However, thorough evaluation of its expression in branchiogenic carcinoma is lacking. The strong positivity in our case demonstrates that human papillomavirus (HPV) is likely to be a tumorigenic agent in branchial cleft primary malignancies as well, and may not be useful in distinguishing from another oropharyngeal primary.

Due to the debate and difficulty in establishing primary disease versus metastasis in the setting of a cystic SCC arising in the neck, evaluative criteria have been proposed to detect additional primaries or metastases. Devaney et al. suggest that when a cystic SCC is found and the consideration of a branchiogenic primary is raised, a series of steps should occur to survey for additional primary sties [11]. The mass must first be proven to be a cystic SCC by imaging, biopsy or fine needle aspiration. Secondly, thorough imaging by CT, MRI and PET/CT should be performed with the addition of direct imaging via panendoscopy. Additionally, bilateral tonsillectomy should occur in concordance with mass resection if imaging methods have not identified a definitive primary site, as the tonsils are a common site of occult primary disease in head and neck SCC [11]. Their recommendations seem to, once more, make the absolute assertion that two separate primaries, particularly one of branchial cleft origin is implausible or impossible.

Treatment of these tumors involves wide local excision with neck dissection and postexcisional radiation therapy $[7,12]$. The addition of carboplatin and 5-flourouracil chemotherapy to the adjuvant radiation therapy has also proven beneficial [13].

Our case represents an exception to the previously established diagnostic criteria for branchiogenic carcinoma. The evolution of secondary primary cancers in head and neck SCC is a well-regarded entity [14]. We propose that histological evidence of branchiogenic carcinoma is the most important diagnostic criterion for this entity and that the presence of another primary should not be viewed as an absolute contraindication to its use as a diagnosis. However, thorough evaluation of the addi- tional primary should be done before giving the diagnosis of two primaries, as the incidence of metastatic involvement of lymph nodes is much more common than branchiogenic carcinoma. Further work is necessary to elucidate the definitive origination of this entity and any molecular peculiarities it may contain. In addition, further studies are necessary to evaluate the role of HPV in branchiogenic carcinoma. We propose that an epithelial lining, compatible with branchial cyst, as well as the progression of normal to dysplastic epithelium with invasion into the cyst wall should be considered the most important diagnostic criteria in situations where a second head and neck primary exists simultaneously. Careful morphologic evaluation as well as immunohistochemical analysis may help in differentiating these primaries.

\section{Acknowledgements}

The authors would like to thank Tracey Bender for her assistance in the preparation of this work.

\section{REFERENCES}

[1] R. Volkman, “Das Tief Branchiogenic Halscarcinom,” Zentralblatt fur Chirurgie, Vol. 9, 1882, p. 49.

[2] H. Martin, H. M. Morfit and H. Ehrlich. "The Case for Branchiogenic Cancer (Malignant Branchioma),” Annals of Surgery, Vol. 132, No. 5, 1950, pp. 867-887. doi:10.1097/00000658-195011000-00002

[3] R. A. Khafif, R. Prichep and S. Minkowitz, "Primary Branchiogenic Carcinoma,” Head \& Neck, Vol. 11, No. 2, 1989, pp. 153-163. doi:10.1002/hed.2880110209

[4] L. D. Thompson and D. K. Heffner, "The Clinical Importance of Cystic Squamous Cell Carcinomas in the Neck: A Study of 136 Cases,” Cancer, Vol. 82, No. 5, 1998, pp. 944-956.

doi:10.1002/(SICI)1097-0142(19980301)82:5<944::AIDCNCR21>3.0.CO;2-\#

[5] B. A. Jereczek-Fossa, C. Casadio, J. Jassem, F. Luzzatto, G. Viale, R. Bruschini, F. Chiesa and R. Orecchia, "Branchiogenic Carcinoma-Conceptual or True Clinico-Pathological Entity?” Cancer Treatment Reviews, Vol. 31, No. 2, 2005, pp. 106-114. doi:10.1016/j.ctrv.2004.12.007

[6] M. Bhanote and G. C. Yang, "Malignant First Branchial Cleft Cysts Presented as Submandibular Abscesses in Fine-Needle Aspiration: Report of Three Cases and Review of Literature,” Diagnostic Cytopathology, Vol. 36, No. 12, 2008, pp. 876-881. doi:10.1002/dc.20920

[7] M. R. Girvigian, A. K. Rechdouni, G. D. Zeger, H. Segall, D. H. Rice and Z. Petrovich, "Squamous Cell Carcinoma Arising in a Second Branchial Cleft Cyst," American Journal of Clinical Oncology, Vol. 27, No. 1, 2004, pp. 96-100. doi:10.1097/01.coc.0000047127.46594.C0

[8] J. P. Roche, M. N. Younes, W. K. Funkhouser and M. C. Weissler, "Branchiogenic Carcinoma of a First Branchial Cleft Cyst,” Otolaryngology_Head and Neck Surgery: 
Official Journal of American Academy of Otolaryngology-Head and Neck Surgery, Vol. 143, No. 1, 2010, pp. 167-168. doi:10.1016/j.otohns.2010.01.031

[9] S. S. Park and C. S. Karmody, "The First Branchial Cleft Carcinoma," Archives of Otolaryngology-Head \& Neck Surgery, Vol. 118, No. 9, 1992, pp. 969-971. doi:10.1001/archotol.1992.01880090085022

[10] R. K. Pai, J. Erickson, N. Pourmand and C. S. Kong, "p16(INK4A) Immunohistochemical Staining May Be Helpful in Distinguishing Branchial Cleft Cysts from Cystic Squamous Cell Carcinomas Originating in the Oropharynx,” Cancer, Vol. 117, No. 2, 2009, pp. 108119.

[11] K. O. Devaney, A. Rinaldo, A. Ferlito, C. E. Silver, J. J. Fagan, P. J. Bradley and C. Suarez, "Squamous Carcinoma Arising in a Branchial Cleft Cyst: Have You Ever Treated One? Will You?” The Journal of Laryngology and Otology, Vol. 122, No. 6, 2008, pp. 547-550.
doi:10.1017/S0022215107001004

[12] Y. C. Lin, S. Y. Fang and R. H. Huang, "Branchiogenic Squamous Cell Carcinoma: A Case Report,” International Journal of Oral and Maxillofacial Surgery, Vol. 33, No. 2, 2004, pp. 209-212. doi:10.1054/ijom.2002.0450

[13] H. Katori, A. Nozawa and M. Tsukuda, "Post-Operative Adjuvant Chemoradiotherapy with Carboplatin and 5Fluorouracil for Primary Branchiogenic Carcinoma,” The Journal of Laryngology and Otology, Vol. 119, No. 6, 2005, pp. 467-469. doi:10.1258/0022215054273241

[14] L. G. Morris, A. G. Sikora, S. G. Patel, R. B. Hayes and I. Ganly, "Second Primary Cancers after an Index Head and Neck Cancer: Subsite-Specific Trends in the Era of Human Papillomavirus-Associated Oropharyngeal Cancer," Journal of Clinical Oncology: Official Journal of the American Society of Clinical Oncology, Vol. 29, No. 6, 2011, pp. 739-746. doi:10.1200/JCO.2010.31.8311 